\title{
CONTROLE DA DOR PÓS-OPERATÓRIA: COMPARAÇÃO ENTRE MÉTODOS ANALGÉSICOS 1
}

Chaves LD, Pimenta CAM. Controle da dor pós-operatória: comparação entre métodos analgésicos. Rev Latino-am Enfermagem 2003 março-abril; 11(2):215-9.

Neste estudo, comparou-se o alívio da dor, a ocorrência de efeitos colaterais, o consumo de analgésicos, regular e complementar, e os custos do tratamento álgico em doentes submetidos a analgesia pós-operatória. Analisaram-se 400 prontuários de pacientes que se submeteram a cinco métodos de analgesia pós-operatória, por via intravenosa e peridural, utilizando ou não bomba de infusão para Analgesia Controlada pelo Paciente (ACP). Foram observadas diferenças entre os métodos, estatisticamente significantes, na intensidade da dor e na ocorrência de efeitos colaterais. No entanto, possivelmente, tais diferenças representaram pequena expressão clínica. Observou-se diferença de custo entre os métodos, e estas foram significativas, o que, talvez, possa ser fator importante na escolha do método analgésico.

DESCRITORES: dor pós-operatória, tratamento da dor, analgesia controlada pelo paciente, custo da analgesia

\section{POSTOPERATIVE PAIN CONTROL: COMPARISON AMONG ANALGESIC METHODS}

This study compared pain relief, the occurrence of side-effects, the regular and supplementary consumption of analgesics, and the costs to treat pain in patients submitted to postoperative analgesia. 400 records were analyzed concerning patients who were submitted to five methods of postoperative analgesia through intravenous and epidural routes, whether or not utilizing infusion pump for Patient-Controlled Analgesia (PCA). Statistically significant differences between the methods were observed as to pain intensity and occurrence of side-effects. However, possibly such differences had low clinical expression. Significant differences were observed as to the cost of the methods, which might be an important factor on choosing an analgesic method.

DESCRIPTORS: postoperative pain, pain treatment, patient-controlled analgesia, analgesia cost

\section{CONTROL DEL DOLOR POST-OPERATORIO: COMPARACIÓN ENTRE LOS MÉTODOS ANALGÉsICOS}

En este estudio, se comparó el alivio del dolor, a la ocurrencia de efectos colaterales, el consumo de analgésicos, regular y complementar y los costos del tratamiento álgido con enfermos sometidos a analgesia post-operatoria. Se analizaron 400 historias clínicas de pacientes que se sometieron a cinco métodos de analgesia post-operatoria por vía intravenosa y peridural, utilizando o no, bomba de infusión para Analgesia Controlada por el Paciente (ACP). Se observaron diferencias entre los métodos estadísticamente significativas, en la intensidad del dolor y en la ocurrencia de efectos colaterales. Aun así, tales diferencias representaron posiblemente una pequeña expresión clínica. Se observaron diferencias de costo entre los métodos, y estas fueron significativas, lo que tal vez pueda ser el factor importante en la elección del método analgésico.

DESCRIPTORES: dolor post-operatorio, tratamiento del dolor, analgesia controlada por el paciente, costo de la analgesia

\footnotetext{
${ }^{1}$ Dissertação de Mestrado apresentada à Escola de Enfermagem da Universidade de São Paulo; ${ }^{2}$ Enfermeira, Mestre em Enfermagem, Professor do Curso de Graduação em Enfermagem da Faculdade Santa Marcelina, e-mail: jbchaves@uol.com.br; ${ }^{3}$ Enfermeira, Professor Livre-Docente da Escola de Enfermagem da Universidade de São Paulo
} 
INTRODUÇÃO

O controle da dor pós-operatória é essencial para a assistência integral ao paciente cirúrgico, visto que estímulos dolorosos prolongados parecem predispor a maior sofrimento e complicações no pós-operatório(1-2), embora estudos recentes, com grupo controle, sejam escassos.

Para o controle da dor pós-operatória, é indicada a abordagem multimodal, que inclui as técnicas farmacológicas e não-farmacológicas. O objetivo é bloquear a geração, transmissão, percepção e apreciação dos estímulos nociceptivos, o que pode ser feito em diferentes níveis do sistema nervoso central e periférico.

Para a abordagem farmacológica, dispõe-se de analgésicos de ação periférica e central, e, também, de anestésicos para uso em bloqueios peridurais e periféricos $^{(3-4)}$. As prescrições de analgésicos devem ser regulares e em esquema, "se necessário". Tal esquema propicia constância do nível plasmático e oferta para episódios de picos de dor ${ }^{(5)}$.

Dentre as principais técnicas não farmacológicas, temos as terapias físicas (aplicação de calor e frio, massagem, estimulação elétrica transcutânea $e$ acupuntura), que, por meio da ativação do sistema sensitivo-discriminativo, estimulam o sistema supressor de dor, e técnicas cognitivo-comportamentais (relaxamento, técnicas de distração, imaginação dirigida, hipnose e biofeedback) que, possivelmente, promovem relaxamento muscular, distração da atenção e sugestão, o que interfere na apreciação do estímulo doloroso pósoperatório $^{(6-7)}$.

Os princípios que norteiam o controle da dor aguda são a seleção de drogas e doses adequadas, a escolha da via e sistema de infusão apropriado, a investigação de possíveis efeitos adversos, a educação do doente, o uso de medidas físicas e comportamentais, a avaliação da eficácia analgésica ${ }^{(8)}$ e da incapacidade oriunda da dor. Tais fatores são, também, a chave para a retroalimentação do sistema.

Os antiinflamatórios não-esteroidais e os opióides são agentes analgésicos que desempenham papel significativo no controle da dor pós-operatória. Atuam em estruturas periféricas e centrais, inibindo a gênese e a condução do estímulo doloroso ${ }^{(2,5,9)}$.

Há que se destacar o desenvolvimento de sistemas de liberação e de infusão de fármacos. O uso de bombas de infusão para a Analgesia Controlada pelo Paciente (ACP) e a analgesia por cateter peridural com opióides, associados ou não a drogas anestésicas, são consideradas técnicas sofisticadas, que envolvem alta tecnologia ${ }^{(3,10-11)}$. No entanto, há duvidas sobre a superioridade desses métodos em relação aos tradicionais (analgesia intravenosa, intramuscular e por via oral), no que se refere ao alívio da dor, à ocorrência de efeitos colaterais, ao número de complicações e ao custo, entre outros $^{(12)}$. Há interesse em se compararem os métodos disponíveis para analgesia, considerando-se que os dados existentes em literatura não são conclusivos, especialmente em nosso meio.

Com intuito de se compararem métodos analgésicos, realizou-se um estudo retrospectivo com doentes submetidos a diferentes protocolos de analgesia pós-operatória, onde se analisou o alívio da dor, a ocorrência de efeitos colaterais, o consumo de analgésico regular e complementar, e o custo direto dos métodos: analgesia peridural e analgesia controlada pelo paciente, nas suas diversas modalidades.

\section{CASUÍSTICA E MÉTODO}

Foram analisados 400 prontuários de pacientes que foram submetidos a tratamento analgésico no pósoperatório, em um hospital geral e privado de São Paulo, no período de janeiro de 1997 a dezembro de 1998. Foram excluídos 18 doentes cujo tratamento analgésico foi interrompido antes do primeiro dia de pós-operatório, período em que se analisaram os dados. Desse modo, a amostra foi composta por 382 prontuários de pacientes. Dos estudados, 332 doentes receberam analgesia por meio de bomba de infusão (BI), com sistema de Analgesia Controlada pelo Paciente (ACP), e 53 doentes não utilizaram bombas de infusão. Dos 332, 139 (36,4\%) receberam analgésicos no modo "intravenoso contínuo, associado a doses em bolo" (IVC+B); 128 (33,5\%), no modo "intravenoso por meio de doses em bolo"(IVB); 41 $(10,7 \%)$ no modo "peridural contínuo associado a doses em bolo"(PC+B) e 21 (5,5\%), no modo "peridural por meio de doses em bolo"(PB). A técnica de "analgesia peridural por meio de doses em bolo - infusão realizada com seringas" (PS), foi realizada em $53(13,9 \%)$ pacientes.

A idade média dos doentes foi 51,6 anos 
(mediana=53,0 e variação entre 8 e 91 anos), e 194 (50,8\%) foram do sexo masculino. Os doentes foram submetidos a cirurgias de variados portes (médio, grande e especial) e tipos (torácica, abdominal, ortopédica, cabeça pescoço, vascular, coloproctológica, ginecológica e urológica).

O prontuário, de modo geral, e o Protocolo do Serviço de Terapia da Dor, de modo especial, foram as bases de dados. Os pacientes foram caracterizados quanto ao sexo, idade, tipo e porte de cirurgia, tipo de técnica analgésica utilizada, via de administração e o modo de infusão. A intensidade da dor, a presença de efeitos colaterais e o consumo de analgésico regular e complementar foram avaliados no $1^{\circ}$ dia de pós-operatório (PO), que correspondeu ao período após a $24^{\mathrm{a}}$ hora até a $48^{a}$ hora após a cirurgia.

Foram excluídas as primeiras 23 horas, pois, nesse período, os pacientes poderiam apresentar alterações cognitivas, que poderiam dificultar o relato verbal; apresentar náuseas, vômitos e retenção urinária, complicações que, talvez, estivessem relacionadas com a anestesia, e ainda estar sob o efeito de drogas anestésicas. Tais fatos poderiam ser fator de confusão na avaliação da efetividade dos protocolos analgésicos.

Os doentes dos 5 diferentes métodos analgésicos foram comparados quanto à intensidade da dor, os efeitos colaterais, o consumo de analgésicos e o custo. Analisouse a homogeneidade dos grupos quanto ao sexo, idade, tipo e porte cirúrgico. Em todas as provas, utilizou-se nível de significância de $5 \%$.

As variáveis dependentes (dor, efeitos colaterais, consumo de analgésicos, dose de opiáceos e custo) foram categorizadas. A intensidade da dor foi classificada em 0 =ausência de dor, $1=$ dor leve, $2=$ dor moderada e $3=$ dor intensa. Os efeitos colaterais foram registrados quanto à freqüência e ao tipo. O consumo de analgésico regular e complementar foi analisado quanto ao número de recebimentos (analgésico complementar) e doses diárias (analgésico regular).

A dose dos analgésicos regular e complementar foi convertida para o equivalente em morfina, e o custo direto de cada método analgésico foi calculado considerando-se a listagem de preços fornecida pelo hospital do estudo (fevereiro de 2000). A Tabela de Procedimentos Médicos da Associação Médica Brasileira (AMB), de 1999, foi utilizada para a identificação da taxa de visita médica e taxa de colocação de cateter peridural. Não foi considerado o custo da hora de trabalho dos profissionais da enfermagem para a administração e manutenção da infusão, por não se dispor de dados que permitissem tal cálculo.

\section{RESULTADOS}

Os grupos não diferiram quanto ao sexo e idade, e diferiram quanto ao porte cirúrgico. Nas cirurgias de grande porte, houve predomínio na utilização de todos os métodos analgésicos. Quando se comparou a utilização dos métodos analgésicos com o porte cirúrgico, observouse, pelo teste Qui-quadrado, diferença entre os grupos $(p=0,004)$. Realizaram-se comparações entre os grupos por meio das partições de Qui-quadrado que demonstraram, no método PS, maior proporção de pacientes submetidos a cirurgias de porte especial que nos demais grupos $(p=0,001)$.

Na Tabela 1, estão apresentadas as médias e medianas da intensidade da dor, por método analgésico.

Tabela 1 - Média e mediana da intensidade média de dor por método analgésico. São Paulo, 2000

\begin{tabular}{lccccc}
\hline & \multicolumn{5}{c}{ Método analgésico } \\
\cline { 2 - 6 } Intensidade & IVB & IVC+B & PB & PC+B & PS \\
média de dor & $\mathbf{n = 1 2 6}$ & $\mathbf{n = 1 2 8}$ & $\mathbf{n = 2 1}$ & $\mathbf{n = 4 0}$ & $\mathbf{n = 5 3}$ \\
\hline Média & 0,43 & 0,55 & 0,35 & 0,24 & 0,43 \\
Mediana & 0,25 & 0,25 & 0,00 & 0,13 & 0,25 \\
Erro padrão & 0,05 & 0,05 & 0,10 & 0,05 & 0,08 \\
Mínimo & 0 & 0 & 0 & 0 & 0 \\
Máximo & 3 & 2,5 & 1,5 & 1,25 & 3
\end{tabular}

ANOVA $(p=0,024)$

Pode-se observar, pela Tabela 1, que, embora tenha existido diferença estatisticamente significante $(p=0,024)$ entre os grupos, a variação entre os valores médios da intensidade da dor foi pequena. Todos os valores foram abaixo de 1, o que correspondeu a "dor leve". O método de comparações múltiplas de Dunnet indicou que essa diferença estatística foi devida à diferença entre os métodos IVC+B e $P C+B(p<0,05)$, que apresentaram, respectivamente, a maior $(0,55)$ e a menor $(0,24)$ média.

$\mathrm{Na}$ Tabela 2, está apresentada a ocorrência de efeitos colaterais, de acordo com o método analgésico. 
Tabela 2 - Freqüência dos efeitos colaterais, segundo o número de doentes por método. São Paulo, 2000

\begin{tabular}{|c|c|c|c|c|c|c|c|c|c|c|c|}
\hline \multirow{4}{*}{$\begin{array}{l}\text { Efeitos } \\
\text { colaterais }\end{array}$} & \multicolumn{10}{|c|}{ Método analgésico } & \multirow{4}{*}{$\begin{array}{c}\text { Teste } \\
\text { Qui- } \\
\text { quadrado } \\
\text { (p) }\end{array}$} \\
\hline & \multicolumn{2}{|c|}{ IVB } & \multicolumn{2}{|c|}{ IVC+B } & \multicolumn{2}{|c|}{ PB } & \multicolumn{2}{|c|}{$\mathrm{PC}+\mathrm{B}$} & \multicolumn{2}{|c|}{ PS } & \\
\hline & $\mathrm{N}$ & $\%$ & $\mathrm{~N}$ & $\%$ & $\mathrm{~N}$ & $\%$ & $\mathrm{~N}$ & $\%$ & $\mathrm{~N}$ & $\%$ & \\
\hline & & & & & & & & & & & \\
\hline Sim & 48 & 37,5 & 57 & 41,0 & 14 & 66,7 & 18 & 43,9 & 34 & 64,2 & 0,003 \\
\hline Não & 80 & 62,5 & 82 & 59,0 & 7 & 33,3 & 23 & 56,1 & 19 & 35,8 & \\
\hline Náusea & 35 & 27,3 & 47 & 33,8 & 8 & 38,1 & 13 & 31,7 & 18 & 34,0 & 0,748 \\
\hline Vômito & 24 & 18,8 & 24 & 17,3 & 6 & 28,6 & 4 & 9,8 & 11 & 20,8 & 0,428 \\
\hline Prurido & 7 & 5,5 & 12 & 8,6 & 3 & 14,3 & 4 & 9,8 & 21 & 39,6 & $<0,001$ \\
\hline $\begin{array}{l}\text { Retenção } \\
\text { urinária }\end{array}$ & 9 & 7,0 & 12 & 8,6 & 5 & 23,8 & 8 & 19,5 & 4 & 7,5 & 0,033 \\
\hline
\end{tabular}

Do total de doentes estudados, 171(44,8\%) apresentaram efeitos colaterais, perfazendo média de 0,72 efeitos por doente. Observou-se diferença estatisticamente significante ( $p=0,003$, Qui-quadrado) entre os métodos. Pela partição de Qui-quadrado, verificou-se que os métodos PB e PS apresentaram mais efeitos colaterais que os demais grupos $(p<0,001)$. Quanto ao tipo de efeito colateral, observou-se que o prurido foi mais freqüente no método PS ( $p<0,001$, Qui-quadrado), e a retenção urinária foi mais freqüente nos métodos $\mathrm{PB}$ ( $\mathrm{p}=0,033$, Qui-quadrado; $p=0,002$, partição de Qui-quadrado) e PC+B ( $p=0,002$, partição de Qui-quadrado).

Para cada doente, calculou-se a média e a mediana do consumo de opióides, em 24 horas, durante todo o período de tratamento (variação entre 1 a 3 dias), o que pode ser observado na Tabela 3.

Tabela 3 - Médias e medianas do consumo de opióide, em 24 horas de tratamento, por método analgésico. São Paulo, 2000

\begin{tabular}{lccccc}
\hline \multirow{2}{*}{$\begin{array}{c}\text { Dose de opióide } \\
\text { em 24h (mg) }\end{array}$} & $\mathrm{n}=69$ & $\mathrm{n}=73$ & $\mathrm{n}=9$ & $\mathrm{n}=15$ & $\mathrm{n}=38$ \\
\cline { 2 - 6 } & 18,98 & 18,00 & 2,28 & 1,15 & 2,07 \\
\hline Média & 17,30 & 15,00 & 0,30 & 0,20 & 2,00 \\
Mediana & 1,16 & 0,75 & 0,90 & 0,53 & 0,15 \\
Erro padrão & 3,50 & 3,80 & 0,00 & 0,00 & 0,10 \\
Mínimo & 58,50 & 34,70 & 6,70 & 6,70 & 4,00 \\
Máximo & & & & & \\
\hline
\end{tabular}

ANOVA $(P<0,001)$

Pela Tabela 3, nota-se que os métodos IVC+B e IVB tiveram o maior consumo de opióide em $24 \mathrm{~h}$ (18,98mg e 18,00mg, respectivamente), e o método $P C+B$ foi o de menor consumo $(1,15 \mathrm{mg})$. As comparações múltiplas pelo método de Dunnet indicaram que não existiu diferença entre o consumo de opióide em 24 horas entre IVB e IVC+B; não existiu diferença entre o consumo de opióide em 24 horas entre $\mathrm{PB}, \mathrm{PC}+\mathrm{B}$ e $\mathrm{PS}$; os pacientes dos métodos IVB e IVC+B receberam maior dose média de opióides em 24 horas do que os do método PB, PC+B e PB.

O uso de analgésicos complementares não diferiu entre os grupos ( $p=0,280$, Qui-quadrado). Considerandose todos os doentes do estudo, $17,3 \%$ não receberam analgésico complementar. Dos que receberam, 66,2\% receberam um analgésico, e 16,5\%, dois analgésicos complementares.

Na Tabela 4, estão apresentados os custos dos cinco métodos analgésicos. Nota-se que o custo dos métodos PC+B e PB foi $68,1 \%$ superior ao IVB e IVC+B, e $93,0 \%$, superior ao PS.

Tabela 4 - Distribuição dos custos diretos por métodos analgésicos. São Paulo, 2001

\begin{tabular}{lr}
\hline M étodos analgésicos & C us to direto \\
\hline P C +B e PB & US $\$ 269,63$ \\
IV C + B e IV B & US $\$ 160,46$ \\
P S & US $\$ 139,31$ \\
\hline
\end{tabular}

\section{DISCUSSÃO}

A queixa dolorosa foi bem controlada em todos os métodos analgésicos. A maior média $(0,55)$ de intensidade de dor foi observada no método IVC+B, e a menor média $(0,24)$, no método $P C+B$. Essas diferenças foram estatisticamente significativas $(p=0,024)$, porém todos se encontravam abaixo de 1 , o que foi considerado "dor leve", possivelmente sem expressão clínica. Discutese que o uso de alta tecnologia no controle da dor pode ser seguido de alívio da dor ou de desconforto pelos métodos $^{(13)}$. Ressalta-se a preocupação em se comprovarem os reais benefícios da técnica de escolha.

Efeitos colaterais ocorreram em $44,8 \%$ dos doentes, perfazendo média de 0,72 por doente. Observouse predomínio de prurido $(p<0,001)$ no método $P S$, e retenção urinária $(p=0,002)$ nos métodos $P B$ e $P C+B$. Esses efeitos adversos são inerentes à técnica e a droga utilizada $^{(14)}$.

O consumo de analgésicos opióides, em regime regular, diferiu quando se compararam vias diferentes (intravenosa versus peridural), o que era esperado, visto 
que, no espaço peridural, as doses são muito inferiores às utilizadas nas vias $\mathrm{IV}^{(15-16)}$. Quando se compararam métodos diferentes, mas que utilizaram a mesma via (IVC+B versus IVB, PC+B versus PB versus PS), não se observaram diferenças estatisticamente significantes quanto à média de consumo de opióides. O número de analgésicos complementares utilizados (AINH) não variou entre os cinco métodos.

O método PS foi o mais barato (US\$139,31) e eqüivaleu a $51,6 \%$ do método mais caro (US\$ 269,63).

O presente estudo é, em nosso meio, um dos primeiros a comparar diversas vias e métodos, utilizando parâmetros clínicos, terapêuticos e financeiros, porém apresenta algumas limitações. Trata-se de estudo retrospectivo, o que, se, por um lado, facilita a análise de grande número de doentes, por outro, limita o pesquisador a trabalhar com os dados já previamente registrados.

Os grupos não foram semelhantes quanto ao porte cirúrgico. A análise de custo envolveu apenas o cálculo do custo direto, o que pode não representar integralmente o custo dos métodos.

\section{REFERÊNCIAS BIBLIOGRÁFICAS}

1. Donovan BD. Patient attitudes to postoperative pain relief. Anaesth Intens Care 1983; 11(2):125-9.

2. Portenoy RK, Kanner RM. Pain management: theory and practice. Philadelphia: FA Davis; 1996.

3. Bonica JJ. The management of pain. $2^{\text {th }}$ ed. Philadelphia/ London: Leal \& Febiger;1990.

4. Kehlet H, Dahl JB. The value of multimodal or balanced analgesia in postoperative pain treatment. Anesth Analg 1993; 77:1048-56.

5. Pimenta CA de M. Aspectos culturais, afetivos e terapêuticos relacionados à dor no câncer. [Tese]. São Paulo (SP): Escola de Enfermagem da USP; 1995.

6. Mc Caferry M, Beebe A. Pain. St. Louis: Mosby; 1989.

7. Pimenta CA de M. Alívio da dor: experiências de enfermagem na utilização de técnicas não farmacológicas. Rev Paul Enfermagem 1990; 9(2):73-7.

8. Sousa FAEF. Dor: o quinto sinal vital. Rev Latino-am Enfermagem 2002 maio-junho; 10(3):446-7.

9. Modig J. Beneficial effects on blood loss in total hip replacement when performed under lumbar epidural anaesthesia versus general anaesthesia: an explanatory study. Acta Chir Scand Suppl 1988; 550:95-103.

10. Cousins MJ. Acute and postoperative pain. In: Wall PD, Melzack R. Textbook of pain. $3^{\text {th }}$ ed. London: Churchill Livingstone;1994. p. 357-86.

11. Ready LB. The treatment of postoperative pain. Proceedings of the $6^{\text {th }}$ World Congress on Pain; 1990 Apr 0106; Adelaide, Australia; 1991.
Outro aspecto a ser destacado é que seria desejável, mas não foi possível, pelas características da amostra, a comparação dos métodos mais "sofisticados" com métodos de analgesia "convencionais", como os que utilizam a via oral e a via intramuscular.

Espera-se que estudos futuros possam corroborar, complementar ou refutar os resultados obtidos.

\section{CONCLUSÃO}

A magnitude da dor foi pequena, e a média de efeitos colaterais por doente foi baixa, para todos os cinco métodos. Observou-se maior ocorrência de prurido no método $\mathrm{PS}$ e de retenção urinária nos métodos $\mathrm{PB}$ e $\mathrm{PC}+\mathrm{B}$. As diferenças de intensidade de dor e de efeitos colaterais observadas entre os métodos, embora estatisticamente significantes, possivelmente, representaram pequena expressão clínica. As diferenças de custo entre os métodos foram significativas e, talvez, possam ser fator importante na escolha da terapia analgésica.

12. Egan KJ, Ready BL. Patient satisfaction with intravenous PCA or epidural morphine. Can J Anaesth 1994; 41(1):6-11. 13. Whedon M, Ferrel BR. Professional and ethical considerations in the use of high-tec pain management. Oncol Nurs Forum 1991; 18(7):1135-43.

14. Romanek RM, Posso I de P. Analgesia controlada pelo paciente no tratamento da dor pós-operatória . Rev Soc Bras Estudo da Dor 2000; 2(2):15-25.

15. Ferrante MF. Opioids. In: Ferrante MF, Vadeboncouer TR. Postoperative pain management. London: Churchill Livingstone; 1995. p. 145-209.

16. Welchew EA, Breen DP. Patient-controlled on-demand epidural fentanyl. A comparison of patient-controlled ondemand fentanyl delivered epidurally or intravenously. Anaesthesia 1991; 46:438-41. 